\title{
Thermodynamics of Black Holes in Anti-de Sitter Space
}

\author{
S. W. Hawking ${ }^{1}$ and Don N. Page ${ }^{2}$ \\ 1 University of Cambridge, Department of Applied Mathematics and Theoretical Physics, Silver \\ Street, Cambridge, England \\ 2 Department of Physics, The Pennsylvania State University, University Park, PA 16802, USA
}

\begin{abstract}
The Einstein equations with a negative cosmological constant admit black hole solutions which are asymptotic to anti-de Sitter space. Like black holes in asymptotically flat space, these solutions have thermodynamic properties including a characteristic temperature and an intrinsic entropy equal to one quarter of the area of the event horizon in Planck units. There are however some important differences from the asymptotically flat case. A black hole in anti-de Sitter space has a minimum temperature which occurs when its size is of the order of the characteristic radius of the anti-de Sitter space. For larger black holes the red-shifted temperature measured at infinity is greater. This means that such black holes have positive specific heat and can be in stable equilibrium with thermal radiation at a fixed temperature. It also implies that the canonical ensemble exists for asymptotically anti-de Sitter space, unlike the case for asymptotically flat space. One can also consider the microcanonical ensemble. One can avoid the problem that arises in asymptotically flat space of having to put the system in a box with unphysical perfectly reflecting walls because the gravitational potential of anti-de Sitter space acts as a box of finite volume.
\end{abstract}

\section{Introduction}

The first indication that black holes have thermodynamic properties came with the discovery that in the classical theory of general relativity the area of the event horizon [1] (or equivalently, the square of the irreducible mass [2]) never decreases. There is an obvious analogy with the second law of thermodynamics with the area of the event horizon playing the role of entropy. There were also analogies to the zeroth and first laws of thermodynamics in which the role of temperature was played by a quantity called the surface gravity $\kappa$ which measured the strength of the gravita ional field at the event horizon [3]. These similarities led Bekenstein [4] to suggest that some multiple of the area of the event horizon, 
measured in Planck units, should be identified as the physical entropy of the black hole. This proposal would lead to inconsistencies and violations of the second law if, as was thought at the time, black holes could absorb particles but could not emit anything. In that case black holes could not be in equilibrium with thermal radiation at any non-zero temperature. However this difficulty was removed when it was discovered that, when quantum effects were taken into account, a black hole would create and emit particles as if it were a hot body with a temperature of $\kappa / 2 \pi$ [5]. It then followed from the first law that the entropy of a black hole was $\frac{1}{4} m_{p}^{2} A$, where $A$ is the area of the event horizon and $m_{p}=G^{-1 / 2}$ is the Planck mass in units in which $\hbar=c=k=1$. A deeper understanding of these thermodynamic properties and a direct derivation of the entropy came with the realization that they were a consequence of the periodicity in the imaginary time coordinate needed to remove the singularities in the Euclidean (i.e. positive-definite) versions of black hole metrics [6-8].

The black holes described above tend asymptotically to flat space. However, one can also have black hole solutions to the Einstein equations with a cosmological constant $\Lambda$ which are asymptotic to de Sitter space (if $\Lambda>0$ ) or to anti-de Sitter space (if $\Lambda<0$ ). The former case has been investigated in [9]. It was found that a black hole in a de Sitter space would emit particles with a temperature determined by the surface gravity of the black hole horizon. However, there was also a cosmological event horizon which was present even in the case of no black hole and which also emitted particles with a temperature determined by its surface gravity. Thermal equilibrium was possible only if these two surface gravities were equal which occurred only in the degenerate case of the Nariai metric [10] which is the analytic continuation of $S^{2} \times S^{2}$.

Anti-de Sitter space has generally been regarded as of little physical interest for two reasons. First, the negative value of $\Lambda$, if interpreted as a vacuum energy, corresponds to negative energy density. Second, anti-de Sitter space has the topology $S^{1} \times R^{3}$, where the $S^{1}$ is timelike. It is therefore periodic in time and contains closed timelike curves. These can be removed by passing to the universal covering space, but this is not globally hyperbolic, that is to say that Cauchy data on a spacelike surface determines the evolution of the system only in a region which is bounded by a null hypersurface called a Cauchy horizon [11]. Thus to specify physics in anti-de Sitter space one has to specify not only the initial configuration but also boundary conditions which describe radiation which comes in from infinity. Nevertheless, despite these difficulties, there have been indications in recent years that anti-de Sitter space may have some physical significance. The first of these was that extended theories of supergravity in which the $O(N)$ group is gauged have anti-de Sitter space as their ground or most symmetric state. The second is that it has been possible to extend Witten's proof for the positive mass theorem $[12]$ to anti-de Sitter space $[13,14]$ and to supergravity $[15,16]$. These results show that asymptotically anti-de Sitter solutions are stable even though the potentials that appear in the theories are unbounded below. We shall therefore consider the quantum mechanical and thermodynamic sroperties of black holes in anti-de Sitter space. The results we shall find are broadly similar to those for black holes in asymptotically flat or de Sitter spaces but there are some important differences. 
Like flat space but unlike de Sitter space, anti-de Sitter space has no natural temperature associated with it. The most symmetric "vacuum state" is therefore not periodic in the imaginary time coordinate though it is periodic in real time. This is true even if one works in the covering space. As in flat space, one can construct thermal states at any temperature $T$ by imposing a periodicity $\beta=T^{-1}$ in imaginary time. The gravitational mass of such a thermal state in flat space would be infinite if it has infinite volume and therefore the state would collapse. Even if one restricted the volume to be finite by putting it in some sort of box, the state would still be unstable to the formation of a black hole, no matter how low the temperature $[17,18]$. Moreover, although a black hole can be in equilibrium with thermal radiation at the same temperature, this equilibrium is unstable if the temperature is held constant: if the black hole were to get a bit more mass, its temperature would go down, the rate of absorption would be greater than the rate of emission and the black hole would continue to grow. This instability means that the canonical ensemble cannot be defined in asymptotically flat space if gravitational effects are included. Instead, one has to use a microcanonical ensemble [17] in which a certain amount of energy is placed in an insulated box though even this is unphysical because one cannot construct a box that will prevent gravitons from escaping. If one ignores this difficulty, one finds that one can have a black hole in stable equilibrium with thermal radiation provided that the energy $E>\left(2^{-21} 3^{-1} 5^{4} \pi^{-2} g m_{p}^{8} V\right)^{1 / 5}$, where $V$ is the volume of the box and $g$ is the effective number of spin states.

In anti-de Sitter space the gravitational potential relative to any origin increases at large spatial distances from the origin. This means that the locally measured temperature of a thermal state decreases and that the total energy of the thermal radiation is finite without any need to put it in a box. In fact the gravitational potential causes "confinement" of nonzero rest mass particles and prevents them from escaping to infinity. Zero rest mass particles can escape to infinity but in a thermal state the incoming and outgoing fluxes at infinity are equal. We find that if the temperature is less than $T_{0}=\frac{1}{2 \pi}(-\Lambda)^{1 / 2}$, thermal radiation is stable against collapse to form a black hole. At temperatures higher than $T_{0}$ there are two values of the mass of a black hole that can be in equilibrium with the thermal radiation. The equilibrium at fixed temperature is unstable for the lower of these masses but is locally stable for the higher one. At $T \gtrsim T_{1}=\frac{1}{\pi}\left(-\frac{\Lambda}{3}\right)^{1 / 2}$, if $|\Lambda| \ll m_{p}^{2}$ as we assume, the configuration with a black hole and thermal radiation has a lower free energy than the configuration with just thermal radiation. At a temperature $T>T_{2}$ $\sim\left(-m_{p}^{2} \Lambda\right)^{1 / 4}$, there is no equilibrium configuration without a black hole.

One can also consider a microcanonical ensemble in which one puts a certain amount of energy into asymptotically anti-de Sitter space. On does not need a box with unphysical walls but one has to impose the boundary condition that the incoming flux of zero rest mass particles at infinity is equal to the outgoing flux. If the energy $E<E_{0} \approx\left(2^{-21} 3^{-1} 5^{4} g m_{p}^{8}\right)^{1 / 5}(-\Lambda / 3)^{-3 / 10}$, the dominant configuration will be that of thermal radiation. If $E_{0}<E<E_{1} \approx 1.314 E_{0}$, there will be a configuration with a black hole and thermal radiation which is locally stable but is 
less probable than thermal radiation alone. If $E_{1}<E<E_{2} \sim m_{p}^{2}(-\Lambda)^{-1 / 2}$, the black hole configuration will be more probable but the pure radiation will still be locally stable. If $E>E_{2}$, the pure radiation configuration will always collapse.

There are also charged and rotating black hole solutions in anti-de Sitter space which contribute to the grand canonical ensemble in which the electric potential and rate of rotation act as chemical potentials for charge and angular momentum respectively. These generalizations behave much as one would expect from the asymptotically flat space case, but one difference is that in anti-de Sitter space one can have a rotating black hole in equilibrium with rotating radiation provided that the angular momentum of the black hole is sufficiently small, whereas in asymptotically flat space such an equilibrium is never possible because the rotational velocity of the radiation would have to exceed that of light at large distances from the black hole.

The plan of this paper is as follows. In Sect. 2 we adopt the Euclidean formulation of quantum theory in anti-de Sitter space. We calculate the Euclidean action of a Schwarzschild-anti-de Sitter solution. We use these results in Sect. 3 to study the canonical ensemble. We find that the black hole has an intrinsic entropy equal to a quarter of the area of the event horizon, as in asymptotically flat space. In Sect. 4 we investigate the microcanonical ensemble.

\section{Euclidean Formulation}

The metric of the covering space of anti-de Sitter space can be written in the static form

$$
\begin{aligned}
d s^{2} & =-V d t^{2}+V^{-1} d r^{2}+r^{2}\left(d \theta^{2}+\sin ^{2} \theta d \varphi^{2}\right) \\
V & =1+\frac{r^{2}}{b^{2}} \\
b & \equiv\left(-\frac{3}{\Lambda}\right)^{1 / 2} .
\end{aligned}
$$

Anti-de Sitter space can be obtained from this metric by identifying $t$ periodically with period $\gamma=2 \pi b$. A timelike geodesic through the origin returns to the origin after a half period $\gamma / 2$. A null geodesic does not return to the origin but escapes to infinity. However one can impose the boundary condition that a zero rest mass particle should also return to the origin after a half period $\gamma / 2$.

The substitution $\tau=i t$ makes the metric (2.1) Euclidean, i.e. positive definite. The most natural and symmetric vacuum state for quantum fields on the anti-de Sitter background is defined by a path integral over field configurations which go to zero at large distances in the Euclidean anti-de Sitter metric. This means that the Green functions will be solutions of elliptic equations in the Euclidean space which vanish at large distances. When analytically continued to the Lorentzian section of anti-de Sitter space, these Green functions will be periodic in $t$ with period $\gamma$. One can also embed anti-de Sitter space conformally into half of the static Einstein universe, that is, into the product of half of the spatial three-sphere sections times the time axis. The anti-de Sitter vacuum state for conformally invariant fields is then the state induced from the natural vacuum state in the 
Einstein universe. The reason that the Green functions are periodic is that particles pass right around the Einstein cylinder and return to their original positions in space after a time $\gamma$.

One can construct thermal states in anti-de Sitter space by periodically identifying the imaginary time coordinate $\tau$ with period $\beta=T^{-1}$. These states will be in thermal equilibrium in the static coordinate system (2.1) with a locally measured temperature

$$
T_{\mathrm{loc}}=\beta^{-1} V^{-1 / 2} .
$$

The local temperature is red-shifted by the gravitational potential and decreases like $r^{-1}$ for $r \gg b$. One would therefore expect the thermal energy density to go down like $r^{-4}$ for zero rest mass particles and faster for particles with rest mass. In the case of conformally invariant particles, one can verify this by taking a thermal state on the Einstein universe and conformally transforming. The resultant energymomentum tensor is

$$
T_{v}^{\mu}=A \delta_{v}^{\mu}+f(T) V^{-2}\left(\delta_{v}^{\mu}-4 \delta_{0}^{\mu} \delta_{v}^{0}\right)
$$

where $f(T)=\frac{\pi^{2}}{90} g T^{4}+O\left(b^{-2} T^{2}\right)$. The first constant term arises from the conformal anomaly and may be regarded as a renormalization of the cosmological constant $\Lambda$. The second term has the form of a perfect fluid with $P=\frac{1}{3} \mu$ and $\mu \propto r^{-4}$ for $r \gg b$. Thus the total energy will be finite.

The Schwarzschild-anti-de Sitter metric has the form (2.1), where now

$$
V=1-\frac{2 M}{m_{p}^{2} r}+\frac{r^{2}}{b^{2}} .
$$

This has a horizon at $r=r_{+}$, where $V\left(r_{+}\right)=0$. The substitution $\tau=i t$ makes the metric positive definite for $r>r_{+}$. The apparent singularity at $r=r_{+}$is just like the singularity at the origin of polar coordinates and can be removed if $\tau$ is regarded as an angular coordinate with period

$$
\beta=\frac{4 \pi b^{2} r_{+}}{b^{2}+3 r_{+}^{2}}
$$

Thus, as in asymptotically flat space, a black hole has a natural temperature associated with it although in this case the locally measured temperature decreases indefinitely the further one is from the black hole. From the formula (2.7) one can see that $\beta$ has a maximum value of $2 \pi 3^{-1 / 2} b$ and therefore $T$ has a minimum value of $T_{0}=(2 \pi)^{-1} 3^{1 / 2} b^{-1}$ when $r_{+}=r_{0}=3^{-1 / 2} b$. For $r_{+}>r_{0}$, the temperature $T$ increases with the mass

$$
M=\frac{1}{2} m_{p}^{2} r_{+}\left(1+\frac{r_{+}^{2}}{b^{2}}\right) .
$$

One can compute the difference between the Euclidean action of the black hole metric and that of anti-de Sitter space identified with the same physical period in imaginary time. The calculation is similar to that in asymptotically flat space [8], 
but in this case the contribution of the surface term is zero. The action comes from the difference in four-volumes of the two metrics and is

$$
I=\frac{\pi m_{p}^{2} r_{+}^{2}\left(b^{2}-r_{+}^{2}\right)}{b^{2}+3 r_{+}^{2}} \text {. }
$$

For small values of $r_{+}$or $M$, this is the same as the flat space result but the action has a maximum when $r_{+}=r_{0}$ and becomes negative when $r_{+}>b$. We shall investigate the physical implications of this formula for the action in the following sections.

\section{The Canonical Ensemble}

The canonical ensemble is defined by a path integral over all matter fields and metrics which tend asymptotically respectively to zero and to anti-de Sitter space identified periodically in $\tau$ with period $\beta$. The dominant contribution to the path integral is expected to come from metrics which are near classical solutions to the Einstein equations. Periodically identified anti-de Sitter space is one of these and we take it to be the zero of action and energy. The path integral over the matter fields and metric fluctuations on the anti-de Sitter background can be regarded as giving the contribution of thermal radiation in anti-de Sitter space to the partition function $Z$. For a conformally invariant field this will be

$$
\log Z=3 \pi^{2} b^{3} \int_{0}^{T} T^{-2} f(T) d T=\frac{\pi^{4}}{90} g(b / \beta)^{3}+O(b / \beta) .
$$

The energy of the thermal radiation will be

$$
\langle E\rangle=-\frac{\partial}{\partial \beta} \log Z=3 \pi^{2} b^{3} f(T) \approx \frac{\pi^{4}}{30} g T^{4} b^{3} .
$$

So far the gravitational effect of thermal radiation has been neglected. One can estimate this by solving the Einstein equations with a $\Lambda$ term for a perfect fluid with $P=\frac{1}{3} \mu$. One finds that solutions exist if the mass of the fluid is less than some critical value $M_{2}$ which can be estimated to be of order $m_{p}^{2} b$. This would correspond to a temperature

$$
T_{2} \sim g^{-1 / 4} m_{p}^{1 / 2} b^{-1 / 2}
$$

Thermal radiation at a temperature greater than $T_{2}$ would not be able to support itself against its self gravity and would collapse to form a black hole.

The Schwarzschild-anti-de Sitter solution is probably the only other nonsingular positive-definite solution of the classical equations that satisfies the periodic boundary conditions. The solution exists only if $\beta \leqq \beta_{0}=2 \pi 3^{-1 / 2} b$, i.e. only for temperatures $T \geqq T_{0}=(2 \pi)^{-1} 3^{1 / 2} b^{-1}$.

The Euclidean action for a black hole solution gives a contribution to $\log Z$ of

$$
\log Z=-I=-\frac{m_{p}^{2} \pi r_{+}^{2}\left(b^{2}-r_{+}^{2}\right)}{b^{2}+3 r_{+}^{2}} .
$$


The expectation value of the energy is

$$
\begin{aligned}
\langle E\rangle & =-\frac{\partial}{\partial \beta} \log Z \\
& =\frac{1}{2} m_{p}^{2} r_{+}\left(1+\frac{r_{+}^{2}}{b^{2}}\right)=M .
\end{aligned}
$$

The entropy is

$$
\begin{aligned}
S & =\beta\langle E\rangle+\log Z=m_{p}^{2} \pi r_{+}^{2} \\
& =\frac{1}{4} m_{p}^{2} A,
\end{aligned}
$$

where $A$ is the area of the event horizon. Thus the relation between entropy and area is the same as in asymptotically flat space. For large $M$,

$$
A \approx 4 \pi\left(2 m_{p}^{-2} b^{2} M\right)^{2 / 3} .
$$

This means that the density of states $N(M)$ for the black hole grows like $\exp \left[\pi\left(2 m_{p} b^{2} M\right)^{2 / 3}\right]$. This is sufficiently slow that the integral defining the partition function

$$
Z=\int N(M) e^{-M / T} d M
$$

converges. This shows that the canonical ensemble in asymptotically anti-de Sitter space is well behaved. In asymptotically flat space the density of black hole states goes as $\exp \left(4 \pi m_{p}^{-2} M^{2}\right)$ and so the canonical ensemble is pathological.

For temperatures $T<T_{0}$, the only possible equilibrium is thermal radiation without a black hole. The free energy is negative and is given by

$$
F=-T \log Z=-\frac{\pi^{4}}{90} g b^{3} T^{4}+O\left(b T^{2}\right)
$$

for conformally invariant fields.

If $T>T_{0}$, there are two possible black hole masses that can be in equilibrium with thermal radiation. The lower of these has negative specific heat $\partial M / \partial T$. It is therefore unstable to decay either into pure thermal radiation or to the larger value of the black hole mass. The lower value of the mass also has positive free energy which means that it is less probable than pure thermal radiation. The higher value of the mass has positive specific heat and is therefore at least locally stable. If

$$
T_{0}<T<T_{1}=(\pi b)^{-1},
$$

the free energy of the black hole is positive so this configuration would reduce its free energy if the black hole evaporated completely. The tunneling probability for this to occur will be of the form

$$
\Gamma=A e^{-B},
$$

where $A$ is some determinant and $B$ is the difference between the actions of the lower and higher mass solutions at the same temperature. If $T \gtrsim T_{1}$, the free energy 
of the higher mass black hole solution will be less than that of pure radiation. The pure radiation will then tend to tunnel to the black hole configuration at the rate given by (3.11), where now $B$ is the action of lower mass solution. Finally, if $T>T_{2}$, the radiation will collapse in a time-scale of order $b$ to the higher mass black hole solution in equilibrium with thermal radiation.

The fluctuations of the metric about the black hole solution can be divided into conformal equivalence classes. In each equivalence class one can pick the metric with constant negative scalar curvature $R=4 \Lambda$. One can then decompose the fluctuations into fluctuations in the conformal factor relative to the metric with $R=4 \Lambda$ (conformal fluctuations) and fluctuations which change the conformal geometry (nonconformal fluctuations) $[19,20]$. As in flat space, the conformal fluctuations reduce the action. One therefore has to rotate the contour of integration for them to the imaginary axis. The nonconformal fluctuations modulo gauge transformations are positive definite for flat space and for anti-de Sitter space. However there is one and only one negative mode for a black hole in asymptotically flat space [21,22]. This negative mode makes the one-loop determinant negative and makes the partition function of the black hole purely imaginary. One can interpret this in two ways. First, it implies that the canonical ensemble in asymptotically flat space is unstable to the formation of black holes with a tunneling probability given by (3.11), where $B$ is the action of a black hole with that temperature [18]. Alternatively, if one uses the micro-canonical ensemble, one has to rotate the contour in the relation between the density of states and the partition function in order to obtain convergence. An imaginary partition function is then necessary to give a real density of states [23].

The Schwarzschild-anti-de Sitter solution has a negative nonconformal mode for small values of $M$ as in the asymptotically flat case. This mode is time independent, spherically symmetric, transverse and traceless [22]. It is non-zero on the horizon and vanishes rapidly at infinity without any nodes. As one increases $M$, a zero mode appears at the value $M_{0}$ that corresponds to the maximum of the action. This zero mode is also time independent, spherically symmetric, transverse and traceless and has no nodes. It must therefore be the negative mode passing through zero. For $M>M_{0}$, there will be no negative nonconformal modes.

The implication of these results for the canonical ensemble is that the lower mass black hole at a given temperature, which has a mass $M<M_{0}$, is unstable but contributes to the tunneling amplitude for the formation or disappearance of black holes. The higher mass black hole, for which $M>M_{0}$, is stable. We shall discuss the implications for the microcanonical ensemble in the next section.

\section{Microcanonical Ensemble}

In the microcanonical ensemble one considers all the states that are possible for a system with energy in the interval $E$ to $E+d E$. One assumes that a system changes from configuration to configuration in an ergodic manner so that the probability of being in a particular configuration is proportional to the number of states that it represents. In the case of asymptotically flat space one has to imagine that the 
system is contained in a box with unphysical walls that will reflect everything including gravitons. However in anti-de Sitter space the gravitational potential has the effect of reflecting back all particles of non-zero rest mass. Zero rest mass particles can escape to infinity though they get infinitely red-shifted. One can impose reflecting boundary conditions at spatial infinity which imply that the incoming and outgoing fluxes are equal [24]. It is therefore possible to consider the microcanonical ensemble in asymptotically anti-de Sitter space without having to invoke unphysical boxes.

One is interested in the density of states $N(E)$. The partition function $Z(\beta)$ is the Laplace transform of $N(E)$,

$$
Z(\beta)=\int_{0}^{\infty} N(E) e^{-\beta E} d E .
$$

Thus $N(E)$ is the inverse Laplace transform

$$
N(E)=\frac{1}{2 \pi i} \int_{-i \infty}^{+i \infty} Z(\beta) e^{\beta E} d \beta .
$$

The contour of integration is taken parallel to the imaginary $\beta$ axis and to the right of any singularities in $Z(\beta)$. Provided that $Z(\beta)$ grows less rapidly than exponentially in $\beta$ for large $\beta$, this ensures that $N(E)=0$ if $E<0$. The positive mass theorem for anti-de Sitter space $[13,14]$ indicates that there should not be any states for negative energies.

Pure thermal radiation will give a contribution to $Z$ of the order of

$$
Z \approx \exp \left(\frac{\pi^{4}}{90} g b^{3} \beta^{-3}\right) \text { for } \beta>\beta_{2}=T_{2}^{-1} .
$$

If $\beta<\beta_{2}$ pure thermal radiation would collapse. The integral for $N(E)$ in Eq. (4.2) will have a saddle point at

$$
\beta \approx\left(\frac{\pi^{4}}{30} g b^{3} E^{-1}\right)^{1 / 4}
$$

The second derivative of the logarithm of the integrand in (4.2) is $\frac{2 \pi^{4}}{15} g b^{3} \beta^{-5}$. Thus the path of steepest descent is parallel to the imaginary axis. This means that $N(E)$ is real and is given approximately by

$$
N(E) \approx \exp \left[\frac{4 \pi}{3}\left(\frac{g b^{3}}{30}\right)^{1 / 4} E^{3 / 4}\right] .
$$

This equation will hold for $E<E_{2} \sim m_{p}^{2} b$ which corresponds to the saddle point at $\beta=\beta_{2}$.

The Euclidean action of a black hole of period $\beta$ is

$$
I_{ \pm}=\frac{\pi}{9} m_{p}^{2} b^{2}\left[1-\frac{2\left(\beta_{0}^{2}-\beta^{2}\right)\left(\beta_{0} \pm \sqrt{\beta_{0}^{2}-\beta^{2}}\right)}{\beta_{0} \beta^{2}}\right] .
$$


The + sign corresponds to the higher mass solution and the - sign to the lower mass solution. They will thus make a contribution of order $e^{-I_{+}}$or $e^{-I-}$ to $Z(\beta)$. The one-loop term about the black hole metrics will contribute a factor of order one or $i \exp \left(\frac{\pi^{4}}{90} g b^{3} \beta^{-3}\right)$ respectively. The factor of $i$ arises in the lower mass case from the negative nonconformal mode. In the higher mass case, if $E>M_{0}$, the stationary phase point in Eq. (4.2) will be at

$$
\beta \approx \frac{4 \pi b^{2} r_{+}}{b^{2}+3 r_{+}^{2}}
$$

where $r_{+}$is the solution of

$$
E=\frac{1}{2} m_{p}^{2} r_{+}\left(1+\frac{r_{+}^{2}}{b^{2}}\right)
$$

The second derivative of the logarithm of the integrand is $T^{2} \partial M / \partial T>0$. Thus the path of steepest descent will be parallel to the imaginary axis and $N(E)$ will be real and given by

$$
\begin{aligned}
N(E) & \approx \exp \left(\pi m_{p}^{2} r_{+}^{2}\right) \\
& \approx \exp \left[\pi\left(2 m_{p} b^{2} E\right)^{2 / 3}\right] \text { for } E \gg M_{0}=3^{-3 / 2} 2 m_{p}^{2} b .
\end{aligned}
$$

In the lower mass case the stationary phase point will be also given by (4.7) and (4.8) if $E \gg E_{0} \sim\left(\mathrm{gm}_{p}^{8} b^{3}\right)^{1 / 5}$ so that thermal radiation makes a negligible contribution. If $E_{0}<E \ll M_{0}$, the stationary phase point will be at the larger root of

$$
E=M+E_{\mathrm{rad}} \approx \frac{m_{p}^{2} \beta}{8 \pi}+\frac{\pi^{4}}{30} g b^{3} \beta^{-4},
$$

where a black hole of energy $M$ is in equilibrium with thermal radiation of energy $E_{\text {rad }}$.

The second derivative of the logarithm of the integrand of (4.2) will be negative at each of these saddle points. Thus the path of steepest decent will be parallel to the real axis. This will introduce a factor of $i$ which will cancel the factor of $i$ arising from the negative nonconformal mode. Thus $N(E)$ will be real and will be given by

$$
N(E) \approx \exp \left[4 \pi m_{p}^{-2} M^{2}+\frac{4 \pi}{3}\left(\frac{g b^{3}}{30}\right)^{1 / 4} E_{\mathrm{rad}}^{3 / 4}\right],
$$

where $M$ and $E_{\text {rad }}$ are the two terms of (4.10) that add up to $E$. If $E<E_{0}$, Eq. (4.10) has no solution for a black hole in equilibrium with radiation, so one obtains only the contribution (4.5) of pure thermal radiation.

We can now estimate the probable configurations for the microcanonical ensemble in different ranges of the energy $E$. If

$$
E<E_{0} \approx\left(2^{-21} 3^{-1} 5^{4} g m_{p}^{8} b^{3}\right)^{1 / 5},
$$

the only locally stable configuration is thermal radiation without a black hole. If

$$
E_{0}<E<E_{1} \approx 1.314 E_{0}
$$


there is also a locally stable configuration with a low mass black hole in equilibrium with thermal radiation. However the pure radiation state is more probable so that although black holes may form from time to time as a result of fluctuations, they will tend to evaporate away by further fluctuations. If

$$
E_{1}<E<E_{2} \sim m_{p}^{2} b,
$$

the pure radiation and the black hole states will be locally stable but the black hole state will be more probable. Finally, if $E_{2}<E$, the only locally stable state will contain a black hole because thermal radiation will collapse. These results are very similar to those for the microcanonical ensemble in a box of volume $\pi^{2} b^{3}$ in asymptotically flat space $[17,25,7,26]$.

Acknowledgement. We are grateful to Gary Gibbons for discussions. D. N. Page was supported in part by NSF Grant PHY-8117464 and by the Nuffield Foundation.

\section{References}

1. Hawking, S.W.: Gravitational radiation from colliding black holes. Phys. Rev. Lett. 26, 1344-1346 (1971)

2. Christodoulou, D.: Reversible and irreversible transformations in black-hole physics. Phys. Rev. Lett. 25, 1596-1597 (1970)

3. Bardeen, J.M., Carter, B., Hawking, S.W.: The four laws of black hole mechanics. Commun. Math. Phys. 31, 161-170 (1973)

4. Bekenstein, J.D.: Black holes and entropy. Phys. Rev. D 7, 2333-2346 (1973)

5. Hawking, S.W.: Particle creation by black holes. Commun. Math. Phys. 43, 199-220 (1975)

6. Hartle, J.B., Hawking, S.W.: Path-integral derivation of black-hole radiance. Phys. Rev. D 13, 2188-2203 (1976)

7. Gibbons, G.W., Perry, M.J.: Black holes and thermal green functions. Proc. R. Soc. London A 358, 467-494 (1978)

8. Gibbons, G.W., Hawking, S.W. : Action integrals and partition functions in quantum gravity. Phys. Rev. D 15, 2752-2756 (1977)

9. Gibbons, G.W., Hawking, S.W.: Cosmological event horizons, thermodynamics, and particle creation. Phys. Rev. D 15, 2738-2751 (1977)

10. Nariai, H.: On some static solutions of Einstein's gravitational field equations in a spherically symmetric case. Sci. Rep. Tôhoku Univ. 34, 160-167 (1950); on a new cosmological solution of Einstein's field equations of gravitation. Sci. Rep. Tôhoku Univ. 35, 62-67 (1951)

11. Hawking, S.W., Ellis, G.F.R.: The large scale structure of space-time. Cambridge: Cambridge University Press 1973

12. Witten, E.: A new proof of the positive energy theorem. Commun. Math. Phys. 80, 381-402 (1981)

13. Abbott, L.F., Deser, S.: Stability of gravity with a cosmological constant. Nucl. Phys. B 195, 76-96 (1982)

14. Gibbons, G.W., Hawking, S.W., Horowitz, G.W., Perry, M.J.: Positive mass theorems for black holes. Commun. Math. Phys. (to appear)

15. Breitenlohner, P., Freedman, D.Z.: Positive energy in anti-de sitter backgrounds and gauged extended supergravity. MIT preprint (1982); stability in gauged extended supergravity. MIT preprint (1982)

16. Gibbons, G.W., Hull, C.M., Warner, N.P.: The stability of gauged supergravity. DAMTP preprint (1982)

17. Hawking, S.W.: Black holes and thermodynamics. Phys. Rev. D 13, 191-197 (1976)

18. Gross, D.J., Perry, M.J., Yaffe, L.G. : Instability of flat space at finite temperature. Phys. Rev. D 25, 330-355 (1982)

19. Gibbons, G.W., Hawking, S.W., Perry, M.J.: Path integrals and the indefiniteness of the gravitational action. Nucl. Phys. B 138, 141-150 (1978) 
20. Gibbons, G.W., Perry, M.J.: Quantizing gravitational instantons. Nucl. Phys. B 146, 90-108 (1978)

21. Page, D.N.: Positive-action conjecture. Phys. Rev. D 18, 2733-2738 (1978)

22. Perry, M.J.: Instabilities in gravity and supergravity. In: Superspace and supergravity: Proceedings of the Nuffield Workshop, Cambridge, June 16 - July 12, 1980. Hawking, S.W., Roček, M. (eds.). Cambridge: Cambridge University Press 1981

23. Hawking, S.W.: Euclidean quantum gravity. In: Recent developments in gravitation: Cargèse 1978. NATO Advanced Study Institutes Series, Series B: Physics, Vol. 44. Lévy, M., Deser, S. (eds.). New York: Plenum Press 1979

24. Avis, S.J., Isham, C.J., Storey, D.: Quantum field theory in anti-de sitter space-time. Phys. Rev. D 18, 3565-3576 (1978)

25. Page, D.N.: Thermodynamic paradoxes. Physics Today 30, 11-15 (1977)

26. Page, D.N.: Black hole formation in a box. Gen. Rel. Grav. 13, 1117-1126 (1981)

Communicated by A. Jaffe

Received August 2, 1982 